\title{
Thermodynamic Geometry of Fractional Statistics
}

\author{
Behrouz Mirza 11 and Hosein Mohammadzadeh 2 \\ Department of Physics, Isfahan University of Technology, Isfahan, 84156-83111, Iran
}

\begin{abstract}
We extend our earlier study about the fractional exclusion statistics to higher dimensions in full physical range and in the non-relativistic and ultra-relativistic limits. Also, two other fractional statistics, namely Gentile and Polychronakos fractional statistics, will be considered and similarities and differences between these statistics will be explored. Thermodynamic geometry suggests that a two dimensional Haldane fractional exclusion gas is more stable than higher dimensional gases. Also, a complete picture of attractive and repulsive statistical interaction of fractional statistics is given. For a special kind of fractional statistics, by considering the singular points of thermodynamic curvature, we find a condensation for a non-pure bosonic system which is similar to the Bose-Einstein condensation and the phase transition temperature will be worked out.
\end{abstract}

PACS number(s): 05.20.-y, 67.10.Fj

\footnotetext{
${ }^{1}$ e-mail: b.mirza@cc.iut.ac.ir

${ }^{2}$ e-mail: h.mohammadzadeh@ph.iut.ac.ir
} 


\section{Introduction}

As a generalization of Bose-Einstein and Fermi-Dirac statistics, fractional statistics has been discussed for many years [1, 2, 3, 4]. The idea of generalized Pauli principle or fractional exclusion statistics was introduced by Haldane [5]. His definition of particles obeying the generalized exclusion statistics with finite dimensional Hilbert space is motivated by such physical examples, as quasi particle in the fractional quantum Hall system and spinons in anti-ferromagnetic spin chains. The parameter governing fractional exclusion statistics in this case is defined by $g=-\frac{\Delta d}{\Delta N}$, where $\Delta d$ is the change in the dimension of the single particle Hilbert space and $\Delta N$ is the change in the number of particles when the size of the system and the boundary conditions are unchanged. By definition, therefore, $g=0$ corresponds to bosons and $g=1$ to fermions. A different concept of fractional statistics, namely, fractional exchange statistics arises when the many body wave function of a system of indistinguishable particles is allowed to acquire an arbitrary phase $e^{i \pi \alpha}$ upon an adiabatic exchange process of two particles. Here, $\alpha$ is the so-called exchange statistical parameter, interpolating between $\alpha=0$ (bosons) and $\alpha=1$ (fermions). Such an exchange produces a nontrivial phase only if the configuration space of the collection of particles under study possesses a multiply connected topological structure. Therefore, fractional exchange statistics is usually restricted to two spatial dimensions and can also be formalized, to some extent, in one spatial dimension. However, fractional exclusion statistics, is based on the structure of the Hilbert space, rather than on the configuration space, of the particle assembly and is, thus, not restricted to $d \leq 2$. In many systems, it is also possible to relate the exclusion statistics parameter, $g$, to the exchange statistics parameter, $\alpha$. The thermodynamic properties of two sorts of fractional statistics have been considered by several authors [6, 7, 8, 9]. Specially, the thermodynamics of the anyon gas - a two dimensional system with fractional statistics - has been the subject of much research [10, 11, 12, 13, 14, 15. More recently the thermodynamic geometry of the anyon gas has been considered [16, 17]. There is also another sort of fractional exclusion statistics, introduced by Polychronakos [18]. It is mentionable that the same distribution function was was derived from collision theory by using the detailed balance hypothesis [19, 20]. Furthermore, Gentile statistics is a generalization of Fermi-Dirac and Bose-Einstein statistics which is based on allowing $p$ $(p \in[1, \infty[)$ particles to occupy the same quantum states [21, 22].

The geometrical structure of the phase space of statistical thermodynamics has been studied and the thermodynamic curvature has already been calculated for some models whose thermodynamics is exactly known, where reviews for these models can be found 
in [23] and [24]. This approach has been utilized to study the thermodynamics of black holes [25, 26, 27, 28, 29, 30]. The thermodynamic curvature of the ideal classical gas is zero and it could be a criterion for statistical interaction of the system [31, 32]. Janyszek and Mrugała worked out the thermodynamic curvature for ideal Fermi and Bose gases and reported that the sign of the thermodynamic curvature is always different for ideal Fermi and Bose gases. It may be shown that the sign of thermodynamic curvature specifies the attractive or repulsive statistical interaction of systems. It has been argued that the scalar curvature could be used to show that fermion gases are more stable than boson gases [33]. Also, phase transition properties of van der Waals gas and some other thermodynamic models have been considered and it has been shown that the singular point of the thermodynamic curvature coincides with the critical point of the system [34, 35]. Recently the thermodynamic curvature of the classical limit of the anyon gas has been worked out [16]. Also, the full physical range thermodynamic curvature of the anyon gas has been obtained by using its factorizable property [17]. However, these properties do not hold in arbitrary dimensions [11, 12, 13].

The outline of this paper is as follows. In Sec. 2, the thermodynamic properties of particles obeying Haldane fractional exclusion (HFE) statistics are summarized and the internal energy and the particle number in an arbitrary dimension $D$ is derived. Also, the same thermodynamic quantities are obtained for Polychronakos fractional exclusion (PFE) statistics and Gentile statistics. In Sec. 3, the thermodynamic geometry of three kinds of fractional statistics is investigated and some interesting properties of these systems are explored. Finally, in Sec. 4, condensation of particles with fractional statistics is explored.

\section{The ideal gas of fractional statistical particles}

\subsection{Haldane fractional exclusion statistics}

Fractional exclusion statistics was introduced as a generalization of Pauli exclusion for fermions. Haldane defined the exclusion statistics parameter, $g$, of a particle by

$$
g=-\frac{d_{N+\Delta N}-d_{N}}{\Delta N}
$$

where $N$ is the number of particles and $d_{N}$ is the dimension of one particle Hilbert space obtained by holding the coordinate of $N-1$ particles fixed [5]. The statistical distribution function, $n(\zeta)$, of an ideal gas of fractional statistical particles with the chemical potential $\mu$ and temperature $T$ has been obtained by $\mathrm{Wu}$ using the Haldane's 
fractional statistics [10],

$$
n(\zeta)=\frac{1}{w\left(e^{(\epsilon-\mu) / k T}\right)+g},
$$

where, the function $w(\zeta)$ satisfies the functional equation

$$
w(\zeta)^{g}[1+w(\zeta)]^{1-g}=\zeta \equiv e^{(\epsilon-\mu) / k T} .
$$

The functional equation for $w(\zeta)$ can be solved analytically only in a few special cases. Equation (3) yields the correct solutions for two familiar cases: bosons $(g=0), w(\zeta)=$ $\zeta-1$ and fermions $(g=1), w(\zeta)=\zeta$. In the thermodynamic limit, the internal energy and particle number of an exclusion gas in a $D$ dimensional box of volume $L^{D}$ with the following dispersion relation

$$
\epsilon=a p^{\sigma}
$$

can be written as

$$
\begin{aligned}
U & =\int_{0}^{\infty} \epsilon n(\zeta) \Omega(\epsilon) d \epsilon, \\
N & =\int_{0}^{\infty} n(\zeta) \Omega(\epsilon) d \epsilon,
\end{aligned}
$$

and $\Omega(\epsilon)$ is the density of the single particle state for the system. Neglecting the spin of particle, the standard form of density of states will be

$$
\Omega(\epsilon)=\frac{A^{D}}{\Gamma\left(\frac{D}{2}\right)} \epsilon^{D / \sigma-1},
$$

where, $\sigma$ takes some values for example $(\sigma=2)$ for non relativistic and $(\sigma=1)$ for ultra relativistic particles and $A=\frac{L \sqrt{\pi}}{a^{1 / \sigma} h}$ is a constant and for simplicity we will set it equal to one $(A=1)$. Obtaining the internal energy and particle number of the ideal exclusion gas for special cases where Eq. (3) could be solved will be straightforward. It is possible to solve this equation using elementary methods for special cases $g=\frac{1}{4}, \frac{1}{3}, \frac{1}{2}, \frac{2}{3}, \frac{3}{4}$, 36]. Also, an analytical explicit series solution for the general values of $g$ has been presented by using Lagrange inversion theorem [37]. It was shown that there is a factorizable property for thermodynamic quantities of a two dimensional HFE gas that indicates we can evaluate the internal energy and particle number of the anyon gas as a composition of the internal energy and particle number of fermion and boson gases, while the particle number of anyon, fermion, and boson gases are the same. Moreover, it has been argued that the factorizable property holds only for two spatial dimensions [13]. Therefore, the 
internal energy and particle number cannot be evaluated analytically for the general value of fractional exclusion parameter in an arbitrary dimension. However, another method is available that enables us to explore the thermodynamic geometry of an ideal gas with fractional statistics in arbitrary $D$ dimensions [38]. First, we change the integrating variable $\epsilon$ to $w$. Using Eq. (3), we will have

$$
\begin{aligned}
\beta(\epsilon-\mu) & =g \ln (w)+(1-g) \ln (w+1) \\
d \epsilon & =\frac{1}{\beta} \frac{w+g}{w(1+w)} d w .
\end{aligned}
$$

We then have

$$
\begin{aligned}
& U=\frac{A^{D}}{\Gamma\left(\frac{D}{2}\right)} \beta^{-\frac{D}{\sigma}-1} \int_{w_{0}}^{\infty} \frac{\left\{\ln \left(z w^{g}(1+w)^{1-g}\right)\right\}^{\frac{D}{\sigma}}}{w(1+w)} d w \\
& N=\frac{A^{D}}{\Gamma\left(\frac{D}{2}\right)} \beta^{-\frac{D}{\sigma}} \int_{w_{0}}^{\infty} \frac{\left\{\ln \left(z w^{g}(1+w)^{1-g}\right)\right\}^{\frac{D}{\sigma}-1}}{w(1+w)} d w
\end{aligned}
$$

where, $w_{0}$ corresponds to the value of $w$ for $\epsilon=0$, which satisfies relation (9)

$$
-\beta \mu=g \ln \left(w_{0}\right)+(1-g) \ln \left(w_{0}+1\right)
$$

and $z=e^{\mu / k T}$ is the fugacity of gas. Therefore, for an arbitrary value of fractional exclusion parameter, $g$, temperature $T=1 / \beta k_{B}$, and fugacity $z$ of the system, the proper value of $w_{0}$ could be evaluated and the value of internal energy and particle number will be determined.

\section{$2.2 \quad$ Gentile statistics}

Gentile generalized the Fermi-Dirac and Bose-Einstein statistics by allowing for the possibility of (no more than) $p$ particles to occupy the same quantum state and then derived the following equation for the distribution function [21, 22, 39]:

$$
n=\frac{1}{e^{(\epsilon-\mu) / k T}-1}-\frac{p+1}{e^{p+1(\epsilon-\mu) / k T}-1},
$$

where, for $p=1$, one obtains the Fermi-Dirac distribution function and when $p$ goes to infinity, the Bose-Einstein distribution function will be acquired. We will set $q=\frac{1}{p}$, then $q=0$ will correspond to the boson and $q=1$ to the fermion case. Then, for $0<q<1$, there is a new kind of fractional statistics, namely Gentile statistics. It is straightforward to obtain the internal energy and particle number of an ideal gas with 
particles obeying Gentile statistics,

$$
\begin{aligned}
U & =A^{D} \frac{\Gamma\left(\frac{D}{\sigma}+1\right)}{\Gamma\left(\frac{D}{2}\right)} \beta^{-\left(\frac{D}{\sigma}+1\right)}\left[L i_{\frac{D}{\sigma}+1}(z)\right. \\
& \left.-\left(\frac{q+1}{q}\right)^{-\frac{D}{\sigma}} L i_{\frac{D}{\sigma}+1}\left(z^{\frac{q+1}{q}}\right)\right], \\
N & =A^{D} \frac{\Gamma\left(\frac{D}{\sigma}\right)}{\Gamma\left(\frac{D}{2}\right)} \beta^{-\left(\frac{D}{\sigma}\right)}\left[L i_{\frac{D}{\sigma}}(z)\right. \\
& \left.-\left(\frac{q+1}{q}\right)^{-\left(\frac{D}{\sigma}-1\right)} L i_{\frac{D}{\sigma}}\left(z^{\frac{q+1}{q}}\right)\right],
\end{aligned}
$$

where, $L i_{n}(x)$ denotes the polylogarithm function.

\subsection{Polychronakos fractional statistics}

Polychronakos suggested another kind of fractional exclusion statistics [18]. It can be thought of as a different realization of the exclusion statistics idea. Unlike the Haldane fractional exclusion statistics, the distribution function has a simple form given by

$$
n=\frac{1}{e^{(\epsilon-\mu) / k T}+\alpha} .
$$

Fermions and bosons correspond to $\alpha=1$ and $\alpha=-1$, respectively, while $\alpha=0$ corresponds to Boltzmann statistics. By substituting $\alpha=2 k-1$, fermions and bosons will correspond to $k=0$ and $k=1$ and Polychronakos fractional statistics corresponds to $0<k<1$. The internal energy and particle number are given by:

$$
\begin{aligned}
U & =A^{D} \frac{\Gamma\left(\frac{D}{\sigma}+1\right)}{\Gamma\left(\frac{D}{2}\right)} \beta^{-\left(\frac{D}{\sigma}+1\right)} \frac{L i_{\frac{D}{\sigma}+1}(z-2 k z)}{1-2 k}, \\
N & =A^{D} \frac{\Gamma\left(\frac{D}{\sigma}\right)}{\Gamma\left(\frac{D}{2}\right)} \beta^{-\left(\frac{D}{\sigma}\right)} \frac{L i_{\frac{D}{\sigma}}(z-2 k z)}{1-2 k} .
\end{aligned}
$$

\section{Thermodynamic curvature of fractional statistic gas}

The geometrical structure of the phase space of statistical thermodynamics was explicitly studied by Gibbs. The geometrical thermodynamics was developed by Rup-

peiner and Wienhold [31, 40]. Ruppeiner geometry is based on the entropy representation, where we denote the extended set of $n+1$ extensive variables of the system by $X=\left(U, N^{1}, \ldots, V, \ldots, N^{r}\right)$, while Weinhold worked in the energy representation 
in which the extended set of $n+1$ extensive variables of the system are denoted by $Y=\left(S, N^{1}, \ldots, V, \ldots, N^{r}\right)[23$. It should be noted that we can work in any thermodynamic potential representation that is the Legendre transform of the entropy or the internal energy. The metric of this representation may be the second derivative of the thermodynamic potential with respect to intensive variables; for example, the thermodynamic potential $\Phi$ which is defined as,

$$
\Phi=\Phi\left(\left\{F^{i}\right\}\right)=\Phi\left(1 / T,-\mu^{1} / T, \ldots, P / T, \ldots,-\mu^{r} / T\right),
$$

$\Phi$ is the Legendre transform of entropy with respect to the extensive parameter, $X^{i}$,

$$
F^{i}=\frac{\partial S}{\partial X^{i}},
$$

and $r$ identify the number of various kind of particles. The metric in this representation is given by

$$
g_{i j}=\frac{\partial^{2} \Phi}{\partial F^{i} \partial F^{j}} .
$$

Janyszek and Mrugała used the partition function to introduce the metric geometry of the parameter space [33],

$$
g_{i j}=\frac{\partial^{2} \ln Z}{\partial \beta^{i} \partial \beta^{j}},
$$

where, $\beta^{i}=F^{i} / k$ and $Z$ is the partition function.

\subsection{Haldane fractional exclusion statistcs}

According to Eq. (8)), the parameter space of an ideal fractional exclusion gas is $(\beta, \gamma)$, where $\beta=1 / k T$ and $\gamma=-\mu / k T$. For computing the thermodynamic metric, we select one of the extended variables as the constant system scale. We will implicitly pick Volume in working with the grand canonical ensemble [33]. We can evaluate the metric elements by the definition of metric in Eq. (17). The metric elements of the 
thermodynamic space of an ideal HFE gas are given by

$$
\begin{aligned}
& G_{\beta \beta}=\frac{\partial^{2} \ln Z}{\partial \beta^{2}}=-\left(\frac{\partial U}{\partial \beta}\right)_{\gamma} \\
& =\frac{-\left(\frac{D}{\sigma}+1\right) \beta^{-\left(\frac{D}{\sigma}+2\right)}}{\Gamma\left(\frac{D}{2}\right)} \int_{w_{0}}^{\infty} \frac{\left\{\ln \left(z w^{g}(1+w)^{1-g}\right)\right\}^{\frac{D}{\sigma}}}{w(1+w)} d w \\
& G_{\beta \gamma}=\frac{\partial^{2} \ln Z}{\partial \gamma \partial \beta}=-\left(\frac{\partial N}{\partial \beta}\right)_{\gamma} \\
& =\frac{-\frac{D}{\sigma} \beta^{-\left(\frac{D}{\sigma}+1\right)}}{\Gamma\left(\frac{D}{2}\right)} \int_{w_{0}}^{\infty} \frac{\left\{\ln \left(z w^{g}(1+w)^{1-g}\right)\right\}^{\frac{D}{\sigma}-1}}{w(1+w)} d w \\
& G_{\gamma \gamma}=\frac{\partial^{2} \ln Z}{\partial \gamma^{2}}=-\left(\frac{\partial N}{\partial \gamma}\right)_{\beta} \\
& =\frac{-\beta^{-\frac{D}{\sigma}}}{\Gamma\left(\frac{D}{2}\right)} \int_{w_{0}}^{\infty} \frac{\left\{\ln \left(z w^{g}(1+w)^{1-g}\right)\right\}^{\frac{D}{\sigma}-1}}{(w+g)^{2}} d w .
\end{aligned}
$$

We note that the value of $w_{0}$, according to Eq. (9), is independent of $\beta$ and depend only on $\gamma$. Therefore, in differentiating with respect to $\beta, w_{0}$ is a constant. To obtain the last equation, Eq. (5) is used and differentiated with respect to $w$. $\frac{\partial w}{\partial \gamma}$ is evaluated from Eq. (9).

$$
\begin{aligned}
& \left(\frac{\partial N}{\partial \gamma}\right)_{\beta}=\frac{\partial N}{\partial w} \frac{\partial w}{\partial \gamma} \\
& =\int_{0}^{\infty} \frac{-\Omega(\epsilon)}{(w+g)^{2}} \frac{e^{(\epsilon-\mu) / k T} w^{g}(1+w)^{1-g}}{w+g} d \epsilon
\end{aligned}
$$

By changing the integrating variable to $w$, the equation will be obtained. We consider a system with two thermodynamic degrees of freedom and, therefore, the dimension of the thermodynamic surface or parameter space is equal to two. Thus, the scalar curvature is given by

$$
R=\frac{2}{\operatorname{det} g} R_{1212}
$$

Janyszek and Mrugała [41] demonstrated that if the metric elements are written purely as the second derivatives of a certain thermodynamic potential, the thermodynamic curvature may then be written in terms of the second and the third derivatives. The sign convention for $R$ is arbitrary, so $R$ may be either negative or positive for any case. Our selected sign convention is the same as that of Janyszek and Mrugała, but different from [23]. In two dimensional thermodynamic spaces, the Ricci scalar is defined by, 


$$
R=\frac{2\left|\begin{array}{ccc}
g_{\beta \beta} & g_{\gamma \gamma} & g_{\beta \gamma} \\
g_{\beta \beta, \beta} & g_{\gamma \gamma, \beta} & g_{\beta \gamma, \beta} \\
g_{\beta \beta, \gamma} & g_{\gamma \gamma, \gamma} & g_{\beta \gamma, \gamma}
\end{array}\right|}{\left|\begin{array}{ll}
g_{\beta \beta} & g_{\beta \gamma} \\
g_{\beta \gamma} & g_{\gamma \gamma}
\end{array}\right|^{2}} .
$$

Using the following equations for an ideal fractional exclusion gas

$$
\begin{aligned}
G_{\beta \beta, \beta} & =\frac{\left(\frac{D}{\sigma}+1\right)\left(\frac{D}{\sigma}+2\right)}{\Gamma\left(\frac{D}{2}\right)} \beta^{-\left(\frac{D}{\sigma}+3\right)} \\
& \times \int_{w_{0}}^{\infty} \frac{\left\{\ln \left(z w^{g}(1+w)^{1-g}\right)\right\}^{\frac{D}{\sigma}}}{w(1+w)} d w \\
G_{\beta \beta, \gamma} & =G_{\beta \gamma, \beta}=\frac{\left(\frac{D}{\sigma}\right)\left(\frac{D}{\sigma}+1\right)}{\Gamma\left(\frac{D}{2}\right)} \beta^{-\left(\frac{D}{\sigma}+2\right)} \\
& \times \int_{w_{0}}^{\infty} \frac{\left\{\ln \left(z w^{g}(1+w)^{1-g}\right)\right\}^{\frac{D}{\sigma}-1}}{w(1+w)} d w, \\
G_{\gamma \gamma, \beta} & =G_{\beta \gamma, \gamma}=\frac{\frac{D}{\sigma}}{\Gamma\left(\frac{D}{2}\right)} \beta^{-\left(\frac{D}{\sigma}+1\right)} \\
& \times \int_{w_{0}}^{\infty} \frac{\left\{\ln \left(z w^{g}(1+w)^{1-g}\right)\right\}^{\frac{D}{\sigma}-1}}{(w+g)^{2}} d w \\
G_{\gamma \gamma, \gamma} & =\frac{1}{\Gamma\left(\frac{D}{2}\right)} \beta^{-\frac{D}{\sigma}} \int_{w_{0}}^{\infty}\{w(w+2)-g(1+2 w)\} \\
& \times \frac{\left\{\ln \left(z w^{g}(1+w)^{1-g}\right)\right\}^{\frac{D}{\sigma}-1}}{(w+g)^{4}} d w .
\end{aligned}
$$

The last equation is obtained by applying the chain rule from Eq. (19) and differentiating with respect to $\gamma$. Now, everything required for evaluating the thermodynamic curvature is available. We will focus on two non-relativistic and ultra-relativistic limits.

\subsubsection{Non-Relativistic Limit $(\sigma=2)$}

Now, we can work out the thermodynamic curvature for an ideal HFE gas in an arbitrary dimension. Specially, we are interested in investigating the behavior of the thermodynamic curvature in three spatial dimensions. The thermodynamic geometry of fractional exclusion gas in two dimensions has completely been considered based on the factorizable property. The thermodynamic curvature for isothermal processes and 
some fixed fugacity values as a function of fractional parameter in the non relativistic limit $(\sigma=2)$ are depicted in Figs. 1 and 2 .

Obviously, the thermodynamic curvature for two dimensional gases based on this method coincides with the results of our previous paper [17]. Also, the result for a three dimensional fractional exclusion gas is similar to those of the two dimensional case. The result is expandable to an arbitrary dimension. In the non-relativistic limit, it seems that in all dimensions and in the high temperature (classical or Boltzmann) limit, the sign of the thermodynamic curvature changes at the same value of fractional parameter $g=0.5$. Therefor, in the high temperature limit and in all dimensions, the fractional exclusion gas is divided in to two cases. For $g<0.5$, the thermodynamic curvature is positive or "Bose-like" and for $g>0.5$, it is negative or "Fermi-like". For $g=0.5$, the ideal HFE gas behaves such as an ideal classical gas. Also, one can find an interesting behavior for $z>1$. The thermodynamic curvature has a maximum point. According to the stability interpretation of the value of the thermodynamic curvature these maximum points should be related to a less stable state of the system. It has been shown [33, 42] that we may consider the thermodynamic curvature as a measure of the stability of the system: The bigger the value of $R$, the less stable is the system. This interpretation of stability measures the looseness of the system to fluctuations and does not refer to the fact that the metric is definitely positive. It is also interesting to note that we can find two different values of $g$ with the same value for the thermodynamic curvature. For some values of $z$, we obtain two values for $g$ with zero curvature, which indicates a duality relation between these points. The thermodynamic curvature of a three dimensional fractional exclusion gas is depicted in Fig. 3 for several values of fractional parameter as a function of fugacity.

It has been shown that, at $\mathrm{T}=0$, the particles of general exclusion statistics exhibit a Fermi surface [6]. One can observes that the thermodynamic curvature of the fractional exclusion gas tends toward the value of an ideal fermion gas for any value of fractional parameter except $g=0$ in the large value of fugacity (low temperature limit) which is consistent with the result in [6]. Also, there is no singularity for any value of fractional parameter except for $g=0$ at $z=1$. Hence, there is no phase transition for $g \neq 0$. It has been shown that there is no phase transition in the Abelian Haldane fractional exclusion gas [37].

We restrict ourselves to the region of $z>1$ and investigate the behavior of the dual points in different dimensions. Figure 4. shows the thermodynamic curvature of the HFE gas in various dimensions and for the same values of the thermodynamic parameter space. 
This figure shows that the maximum point goes to the lower value of fractional parameter in the higher dimension. Also, the dual points go away from each other at higher dimensions. Duality may also disappear at lower dimensions. The gas is more stable at higher dimensions. Also, we can see from Fig. 5 that for a fixed dimension of space, the dual points are fixed for different values of temperature. Generally, the sign of the thermodynamic curvature is independent of $\beta$. In other words, we can find a threshold value for fugacity $\left(z_{0}\right)$ for which the thermodynamic curvature in $z>z_{0}$ will be negative for any value of $\beta$.

\subsubsection{Ultrarelativistic Limit $(\sigma=1)$}

In this section, we consider the fractional exclusion particle in the ultra-relativistic limit. First, we represent the result in two spatial dimensions as in Fig 6.

One can perceive that in the classical limit (small value of fugacity), the thermodynamic curvature is zero at $g=0.5$, which is similar to the non-relativistic limit. Also, the general behavior of the thermodynamic curvature in all its physical range is similar to its non-relativistic limit.

The thermodynamic curvature as a function of fugacity and for various values of fractional parameter is depicted in Fig. 7. It shows that the thermodynamic curvature of the boson gas in the ultra-relativistic limit in 2D starts from a positive value in the classical limit and tends to infinity at $z=1$. For small values of fractional parameter, the thermodynamic curvature has a maximum and, finally, goes to the negative values but for large values of the fractional parameter, it is a monotonically decreasing function with respect to $z$. A comparison between the non-relativistic and ultra-relativistic limits shows that the general behavior of the thermodynamic curvature remains the same. Therefore, it seems that the dispersion relation does not affect the general behavior of the thermodynamic curvature and, thereby, the stability of the gas in different dimensions.

\subsection{Gentile statistics}

The thermodynamic geometry of an ideal gentileonic gas can be calculated by using the internal energy and the particle number of the gas. The thermodynamic parameter space is a two dimensional space with the parameters $(\beta, \gamma)$. Therefore, one can easily evaluate the metric elements and their derivatives with respect to the parameters of the thermodynamic space. Thus, we work out the thermodynamic curvature as a function of thermodynamic parameters and the fraction parameter $q$. The thermodynamic 
curvature of ideal gases obeying Gentile's statistics has been investigated by Oshima et al. 433. Here, we can consider it in an arbitrary dimension and for any value of fractional parameter in the full physical range. The thermodynamic curvature is depicted in Fig. 8 as a function of fractional parameter, $q$, for isotherm processes and various values of fugacity, where we suppose $D=3$ and $\sigma=2$. It is clear that in the classical limit (small values of fugacity), and in a vast domain of the fractional parameter, the thermodynamic curvature is mostly positive and less stable than the HFE gas. Zeroth point of the ricci scalar goes to the lowest value by increasing the fugacity. Like the case with the HFE gas for $z>1$, a maximum point appears and for some values of the thermodynamic parameter space, we obtain two values for $q$ with zero curvature, which indicates a duality relation between these points.

Figure 9, shows that for any value of the fraction parameter, and for large value of fugacity (low temperature limit), the thermodynamic curvature tends toward a negative value. Therefore, the particles obeying Gentile statistics, at $T=0$, exhibit a Fermi surfaces.

\subsection{Polychronakos statistics}

Constructing the thermodynamic geometry of an ideal gas with particles obeying Polychronakos fractional exclusion statistics is entirely similar to the previous examples. Using Eq. (13), we can obtain the metric elements and the Ricci scalar curvature in an arbitrary dimension. We will focus on three spatial dimensions. The thermodynamic curvature is depicted as a function of the fractional parameter, $k$, for isotherm processes in Fig 10. Various values have been selected in each curve for fugacity from the classical limit to the quantum limit. It is obvious that in the high temperature limit, the two kinds of fractional exclusion statistics, namely Haldane and Polychronakos fractional exclusion statistics, are the same. In all the physical range, $k=0.5$ coincides with the zeroth point of the thermodynamic curvature. It is easy to show that the distribution function of Polychronakos fractional statistics at $k=0.5$ is the Boltzmann distribution function. Therefore, an ideal fractional exclusion gas at $k=0.5$ is an ideal classical gas, which has zero curvature in the full physical range. Also, for the full physical range, the thermodynamic curvature is positive for $k<0.5$ but negative for $k>0.5$.

It has been shown that the thermodynamic curvature of a boson gas is singular at $z=1$, where the Bose-Einstein condensation occurs. This is clear from Figs. 10 and 11 in which the thermodynamic curvature goes to infinity for $k=0$ (bosons). However, an interesting new phenomenon is observable for $(0<k<0.5)$. For a certain value of 
the fractional parameter in this interval, there is a specific value of fugacity, at which the thermodynamic curvature has a singularity.

\section{Condensation for Particles with Fractional Statis- tics}

Condensation of particles obeying Polychronakos fractional statistics for different values of the fractional parameter is an interesting subject. This kind of condensation $(R=\infty)$ appears for non-pure bosonic particles. In fact, the singularity of the thermodynamic curvature arises from the definition of the polylogarithm function $\left(L i_{n}(x)\right)$, where $x$ has to belong to the interval ] $-\infty, 1]$. Thereupon, one can shows from the thermodynamic quantities such as internal energy and particle number that where $z-2 k z=1$, the thermodynamic curvature face with a singularity. Therefore, for a given value of the fractional parameter, $k$, the singularity occurs at

$$
z_{c}=\frac{1}{1-2 k}
$$

In fact, it indicates a condensation similar to the Bose-Einstein but for a non-zero fractional parameter or a non-pure bosonic system of the fractional gas. Thermodynamic curvature for boson gas and fractional exclusion statistic gas is a function with respect to $\beta$ and $z$ in the following general form

$$
R=\beta^{\frac{D}{\sigma}} f(z, k, D, \sigma)
$$

If we set $z=1$ (boson case) and $z=1 /(1-2 k)$ (Polychronakos statistics), the thermodynamic curvature goes to infinity independent of $\beta$. Therefore, the thermodynamic curvature diverges at critical fugacity for all value of $\beta$. For obtaining the critical temperature we need an additional relation such as the particle number. By substituting $a=\frac{1}{2 m}$ and $\sigma=2$, Eq. (13) yields

$$
n \lambda^{3}(1-2 k)=L i_{\frac{3}{2}}(z-2 k z)
$$

where, $n=\frac{N}{L^{3}}$ and $\lambda=\sqrt{\frac{h^{2}}{2 m \pi k_{B} T}}$ is the thermal wavelength. For a given value of $k$, there is a suitable value for fugacity that satisfies Eq. (23) and $L i_{\frac{3}{2}}(1)=\zeta\left(\frac{3}{2}\right)$ has a finite value. However, it is obvious from Fig. 12 that for the values $k=\frac{1}{4}, \frac{1}{3}, \frac{3}{8}, \cdots$, the function $n \lambda^{3}(1-2 k)$ with an infinite slope at $z=2,3,4, \ldots$ which is similar to an ideal 
boson gas that has infinite slope at $z=1$ where the Bose-Einstein condensation occurs 44.

In constant $n$, we can introduce a critical temperature from Eq. (25),

$$
k_{B} T_{c}=\frac{h^{2}}{2 m \pi}\left[\frac{n(1-2 k)}{\zeta(3 / 2)}\right]^{2 / 3}
$$

where, $\zeta(x)$ is the Riemann zeta function. Also, one can find a restricting condition on the chemical potential of particles with the fraction parameter $k$. Whereas $z$ has to satisfy the inequality $z \leq \frac{1}{1-2 k}$, we obtain

$$
\mu_{k} \leq-k_{B} T \ln (1-2 k)
$$

We can find the transition temperature in an arbitrary dimension and with a general dispersion relation,

$$
n \Lambda^{\frac{D}{\sigma}}(1-2 k)=\frac{\Gamma\left(\frac{D}{\sigma}\right)}{\Gamma\left(\frac{D}{2}\right)} L i_{\frac{D}{\sigma}}(z-2 k z),
$$

where, $n=\frac{N}{L^{D}}$ is the density of the particles in an arbitrary dimension and $\Lambda=\frac{a h^{\sigma}}{\pi^{\frac{\sigma}{2}} k_{B} T}$. Now, similar to the 3D case, condensation occurs for $z=\frac{1}{1-2 k}$. Thus, the transition temperature in constant $n$ is given by

$$
k_{B} T_{c}=\frac{a h^{\sigma}}{\pi^{\sigma / 2}}\left(\frac{n(1-2 k) \Gamma\left(\frac{D}{2}\right)}{\Gamma\left(\frac{D}{\sigma}\right) \zeta\left(\frac{D}{\sigma}\right)}\right)^{\sigma / D} .
$$

According to the zeta function properties, it is obvious that there is a finite transition temperature for $\frac{D}{\sigma}>1$. Furthermore, we notice that in all thermodynamic quantities and all metric elements of the thermodynamic geometry, the dependency on $D$ and $\sigma$ appears as $\frac{D}{\sigma}$. Therefore, the thermodynamic properties such as critical properties for $D=2 n$ dimensional fractional statistic gas in the non-relativistic limit are similar to the $D=n$ dimensional one in the ultra-relativistic limit [45]. In other words, condensation occurs in $D \geq 3$ in the non-relativistic limit but in $D \geq 2$ in the ultra-relativistic limit.

\section{Conclusion}

The internal energy and particle number of three kinds of fractional statistics were represented in an arbitrary dimension. The thermodynamic geometry of an ideal Haldane, Gentile and Polychronakos fractional exclusion statistic gas was also explored in 
arbitrary dimensions. Finally, the thermodynamic curvature of Haldane statistics was worked out in the non-relativistic and ultra-relativistic limits in $2 \mathrm{D}$ and $3 \mathrm{D}$. It was found that in the non-relativistic limit, the results for 3D are similar to those in the 2D. However, moving to higher dimensions, the thermodynamic geometry suggested that less stable gases would be obtained. It was also found that no singularity exists for any value of the fractional parameter, except for $g=0$ at $z=1$, which corresponds to the Bose-Einstein condensation.

The thermodynamic geometry of an ideal gas obeying Gentile statistic was considered. The thermodynamic curvature in the classical limit was found to be mostly positive, which may be related to the attractive statistical interaction of Gentileons. Moving from the classical limit to the larger values of fugacity at $z>1$, one can observe a similarity between the HFE gas and the Gentileonic gas. For some values of thermodynamic parameters, there is a duality between two different values of the fractional parameter in both cases. Also, it seems that at $T=0$, particles obeying the HFE statistics and Gentile statistics exhibit a Fermi surfaces and the repulsive statistical interaction is dominant in the low temperature limit. Again, only the singular point in a gentileonic gas is related to $q=0$ (boson) at $z=1$. In general, the HFE gas is more stable than the Gentileonic gas.

Some special phenomena were found to occur for an ideal Polychronakos fractional exclusion gas. One can find that in the classical limit, two different definitions of the fractional exclusion statistics, namely Haldane and Polychronakos, coincide. It is mentionable that these statistics are different completely in full physical range. Specially, in low temperature limit the exclusion property of particles obeying Haldane statistics is dominant. Therefore, BEC does not occur for particles obeying HFE statistics. In other words, Polychronakos statistics does not drive from Haldane statistics, but these statistics coincide with together in the classical limit. Going far away from the classical limit, their differences begin to appear. The thermodynamic geometry of a PFE gas can be divided into two different regions. For $k<0.5$, the thermodynamic curvature is positive (Bose-like), while for $k>0.5$, it is negative (Fermi-like). Also, there are some singular points of thermodynamic curvature for $k<0.5$ (Bose-like) which correspond to the condensation of the fractional statistics particles. Thus, we find a condensation for a non-pure bosonic system. In all kinds of fractional statistics, a non-relativistic $2 d$-dimensional fractional statistics gas has the same thermodynamic geometry that an ultra-relativistic gas in $d$-dimensions. 


\section{References}

[1] J. M. Leinaas and J. Myrheim, Nuovo Cimento B37, 1 (1977).

[2] G. A. Goldin, R. Menikoff and D. H. Sharp, J. Math. Phys. 21, 650 (1980).

[3] F. Wilczek, Phys. Rev. Lett. 49, 957 (1982).

[4] M. V. Medvedev. Phys. Rev. Lett. 78, 4147 (1997).

[5] F.D.M. Haldane, Phys. Rev. Lett. 67, 937 (1991).

[6] C. Nayak and F. Wilczek, Phys. Rev. Lett. 73, 2740 (1994).

[7] M. V. N. Murthy and R. Shankar, Phys Rev. Lett. 72, 3629 (1994).

[8] M. V. N. Murthy and R. Shankar, Phys Rev. Lett. 73, 3331 (1994).

[9] F. Wilczek, Phys. Rev. Lett 48, 1144 (1982).

[10] Y.-S. Wu, Phys. Rev. Lett. 73, 922 (1994).

[11] W- H. Huang, Phys. Rev. E 51, 3729 (1995).

[12] W- H. Huang, Phys. Rev. B 53, 15842 (1996).

[13] W- H. Huang, Phys. Rev. Lett. 81, 2392 (1998).

[14] F. M. D. Pellegrino, G. G. N. Angilella, N. H. March and R. Pucci, Phys. Rev. E 76, 061123 (2007).

[15] M. T. Batchelor and X.-W. Guan, Phys. Rev. B 74, 195121 (2006).

[16] B. Mirza and H. Mohammadzadeh, Phys. Rev. E 78, 021127 (2008).

[17] B. Mirza and H. Mohammadzadeh, Phys. Rev. E 80, 011132 (2009).

[18] A.P. Polychronakos, Phys. Lett. B 365, 202(1996).

[19] N. H. March, N. Gidopoulos, A. K. Theophilou, M. J. Lea and W. Sung, Phys. Chem. Liq. 26, 135 (1993).

[20] N. H. March, J. Phys.: Condens. Matter 5, B149 (1993).

[21] G. Gentile, Nuovo Cimento 17493 (1940). 
[22] G. Gentile, Nuovo Cimento 19109 (1942).

[23] G. Ruppeiner, Rev. Mod. Phys. 67, 605 (1995).

[24] D. C. Brody and D. W. Hook, J. Phys. A: Math. Theor. 42, 023001 (2009).

[25] J. E. Aman, I. Bengtsson, N. Pidokrajt, Gen. Rel. Grav. 35, 1733 (2003)

[26] B. Mirza and M. Zamani-Nasab, J. High Energy Phys. 06, 059 (2007).

[27] G. Ruppeiner, Phys. Rev. D 78, 024016 (2008).

[28] J. L. Alvarez, H. Quevedo and A. Sanchez, Phys. Rev. D 77, 084004 (2008).

[29] A. Sahay, T.Sarkar, G. Sengupta, arXiv:1004.1625[hep-th].

[30] R. Banerjee, S. Kumar Modak, S. Samanta, arXiv:1005.4832[hep-th].

[31] G. Ruppeiner, Phys. Rev. A 20, 1608 (1979).

[32] J. D. Nulton and P. Salamon, Phys. Rev. A 31, 2520 (1985).

[33] H. Janyszek and R. Mrugała, J. Phys. A 23, 467 (1990).

[34] D. Brody and N. Rivier, Phys. Rev E 51, 1006 (1995).

[35] W. Janke, D. A. Johnston and R. Kenna, Physica A 336, 181 (2004).

[36] T. Aoyama, Eur. Phys. J. B 20, 1, 123-131 (2001).

[37] G. S. Joyce, S. Sarkar, J. Spałek, and K. Byczuk, Phys. Rev. B 53, 990 (1996).

[38] G. G. Potter, G. Müller and M. Karbach, Phys. Rev. E 75, 061120 (2007).

[39] A. Khare, Fractional Statistics and Quantum Theory, World Scientific, Singapore, (1997).

[40] F. Weinhold, J. Chem. Phys. 63, 2479 (1975).

[41] H. Janyszek and R. Mrugała, Phys. Rev. A 39, 6515 (1989).

[42] H. Janyszek and R. Mrugała, J. Phys. A: Math. Gen. 23, 477-490 (1990).

[43] H. Oshima, T. Obata and H. Hara, J. Phys. A: Math. Gen. 32, 6373 (1999).

[44] K. Huang, Statistical Mechanics, John Wiley \& Sons, Inc., New York, (1987).

[45] R. M. May, Phys. Rev. 135, A1515 (1964). 


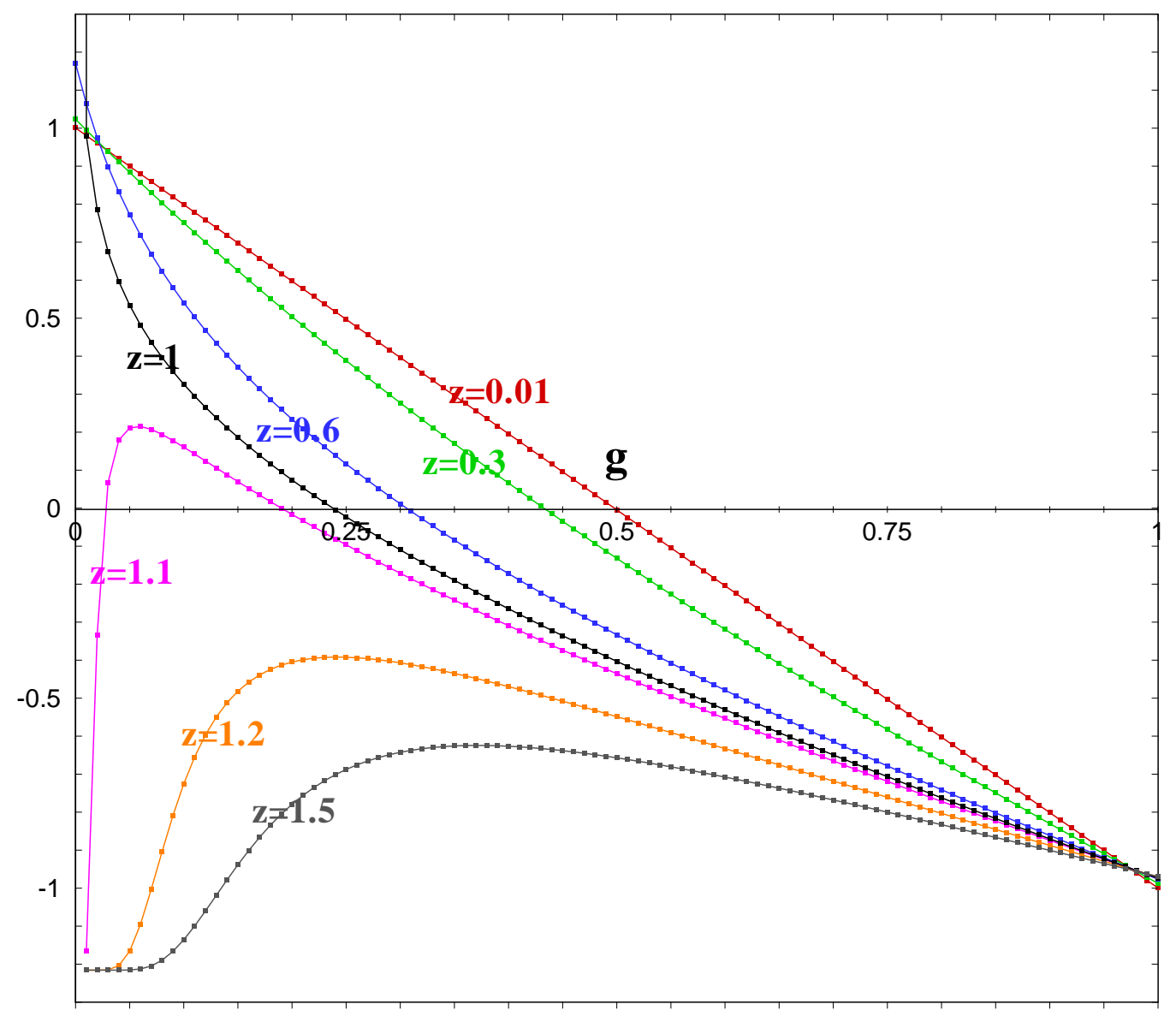

Figure 1: (Color online) The thermodynamic curvature of a 2D HFE gas (nonrelativistic) with respect to the fractional parameter, $\mathrm{g}$, for an isotherm $(\beta=1)$. The assumed values of anyon fugacity are $z=0.01$ [red (upper) line], 0.3 [green line], 0.6 (blue line), 1 [black (middle) line], 1.1 (purple line), 1.2 (orange line), 1.5 [light gray (lower) line]. 


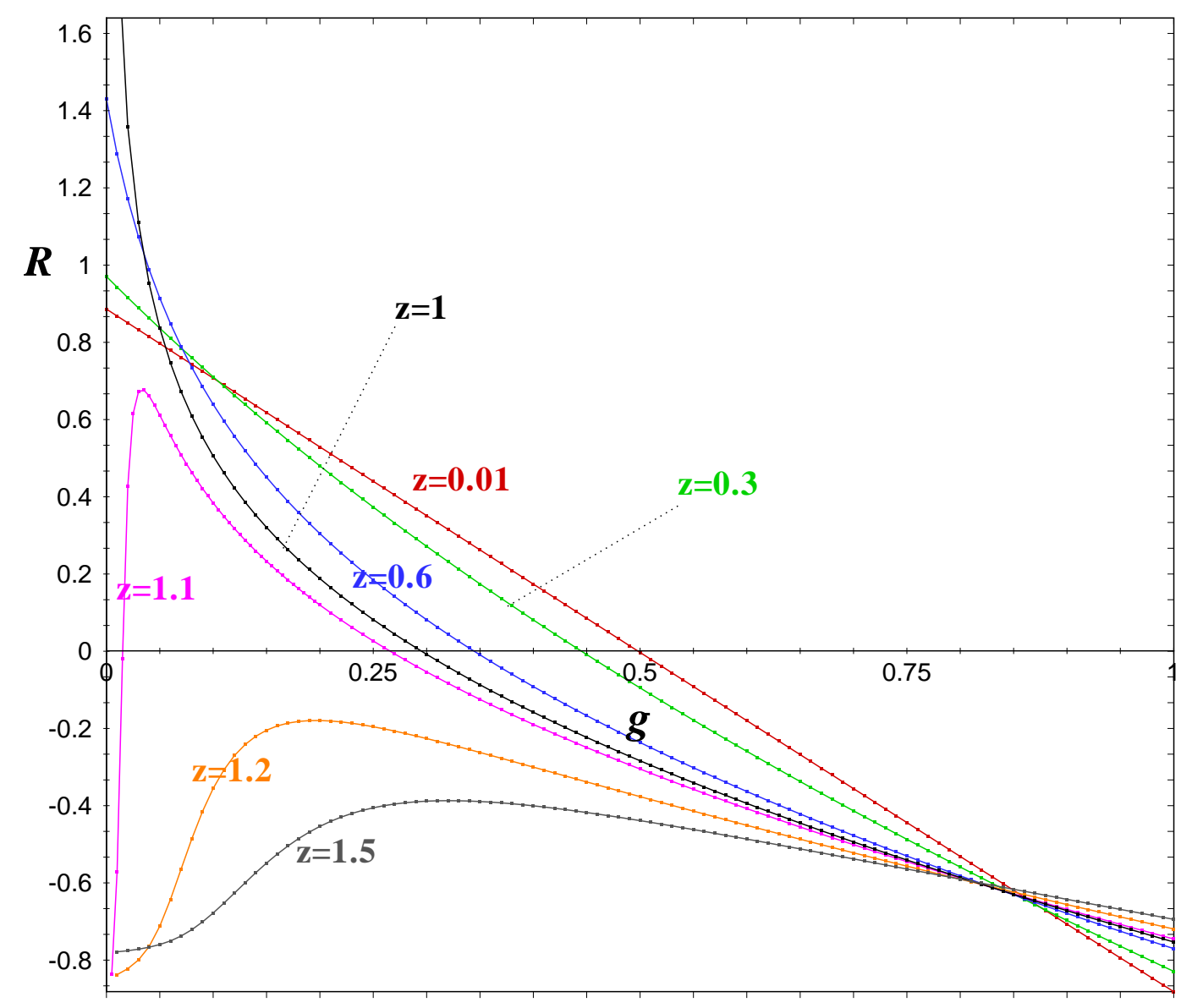

Figure 2: (Color online) The thermodynamic curvature of a 3D HFE gas with respect to the fractional parameter for an isotherm $(\beta=1)$. The assumed values of fugacity are $z=0.01$ [red (upper) line], 0.3 [green line], 0.6 (blue line), 1 [black (middle) line], 1.1 (purple line), 1.2 (orange line), 1.5 [light gray (lower) line]. 


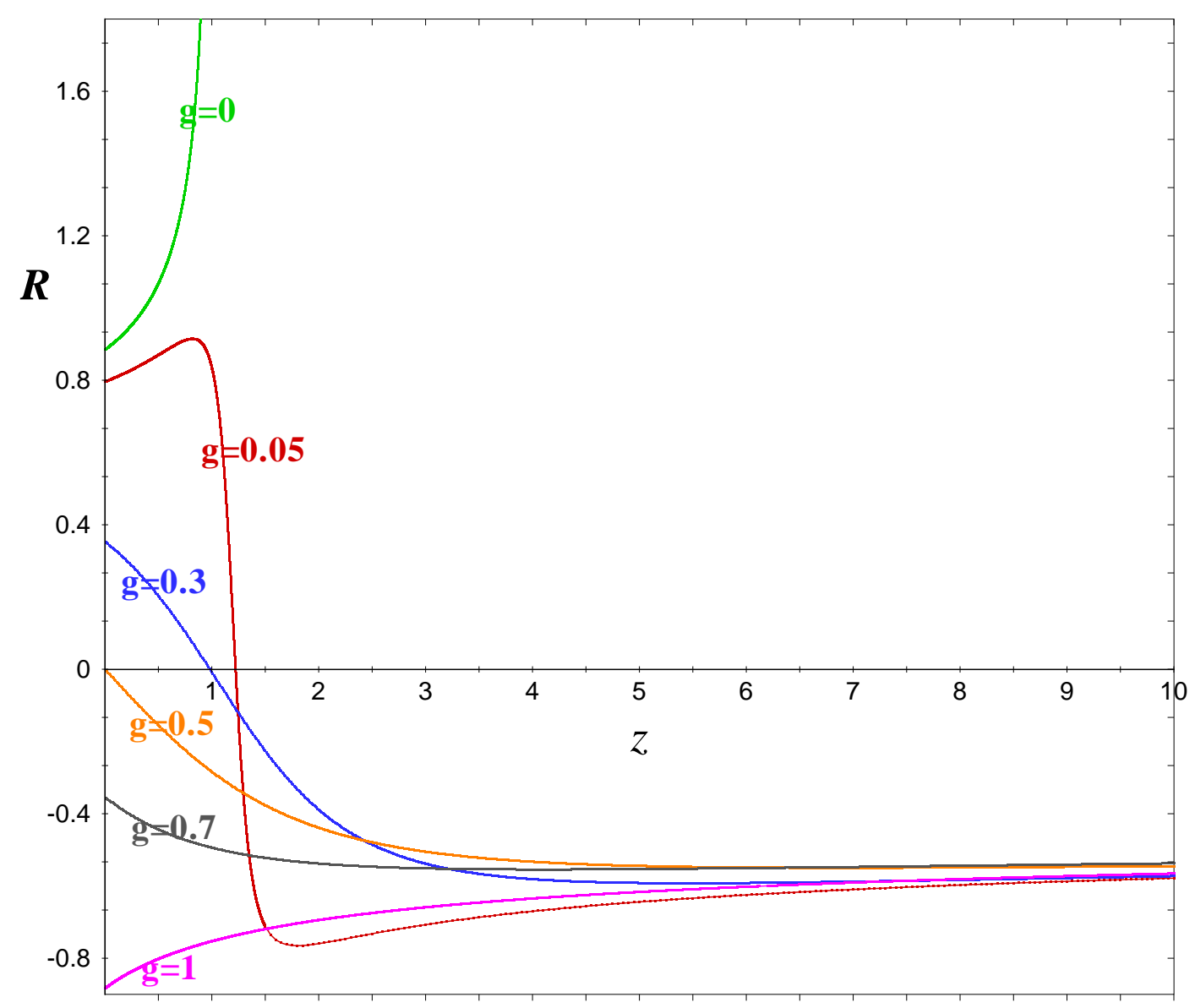

Figure 3: (Color online) The thermodynamic curvature of an ideal 3D HFE gas as a function of fugacity for an isotherm process $(\beta=1)$. The assumed values of the fractional parameter are $g=0$ [green (upper) line], 0.05 (red line), 0.3 (blue line), 0.5 (orange line), 0.7 (black line), 1 [purple (lower) line]. 


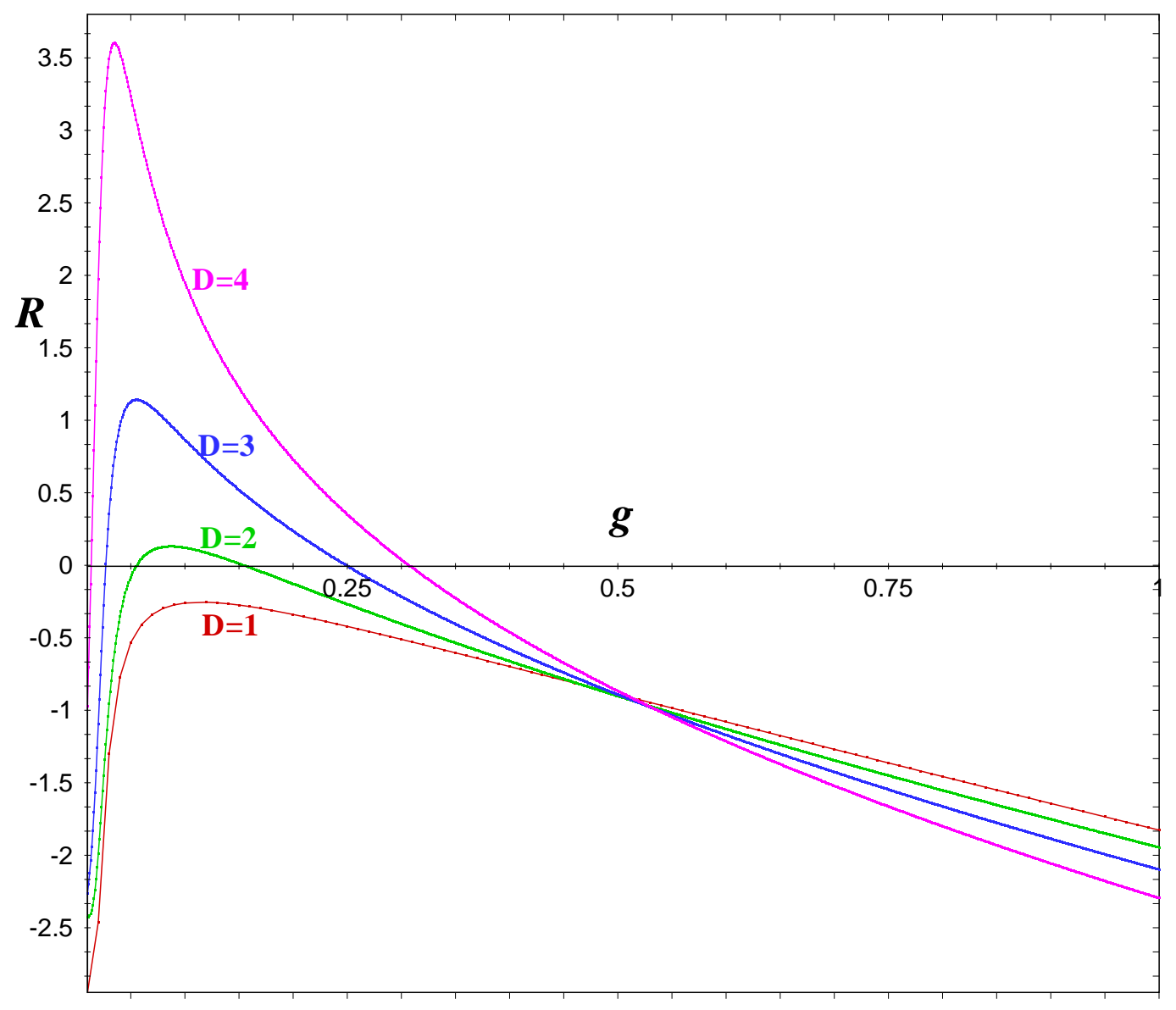

Figure 4: (Color online) The thermodynamic curvature of an ideal HFE gas as a function of the fractional parameter for various dimensions $D=1,2,3,4$ at a fixed point of the thermodynamic parameters space $\beta=2$ and $z=1.15$. 


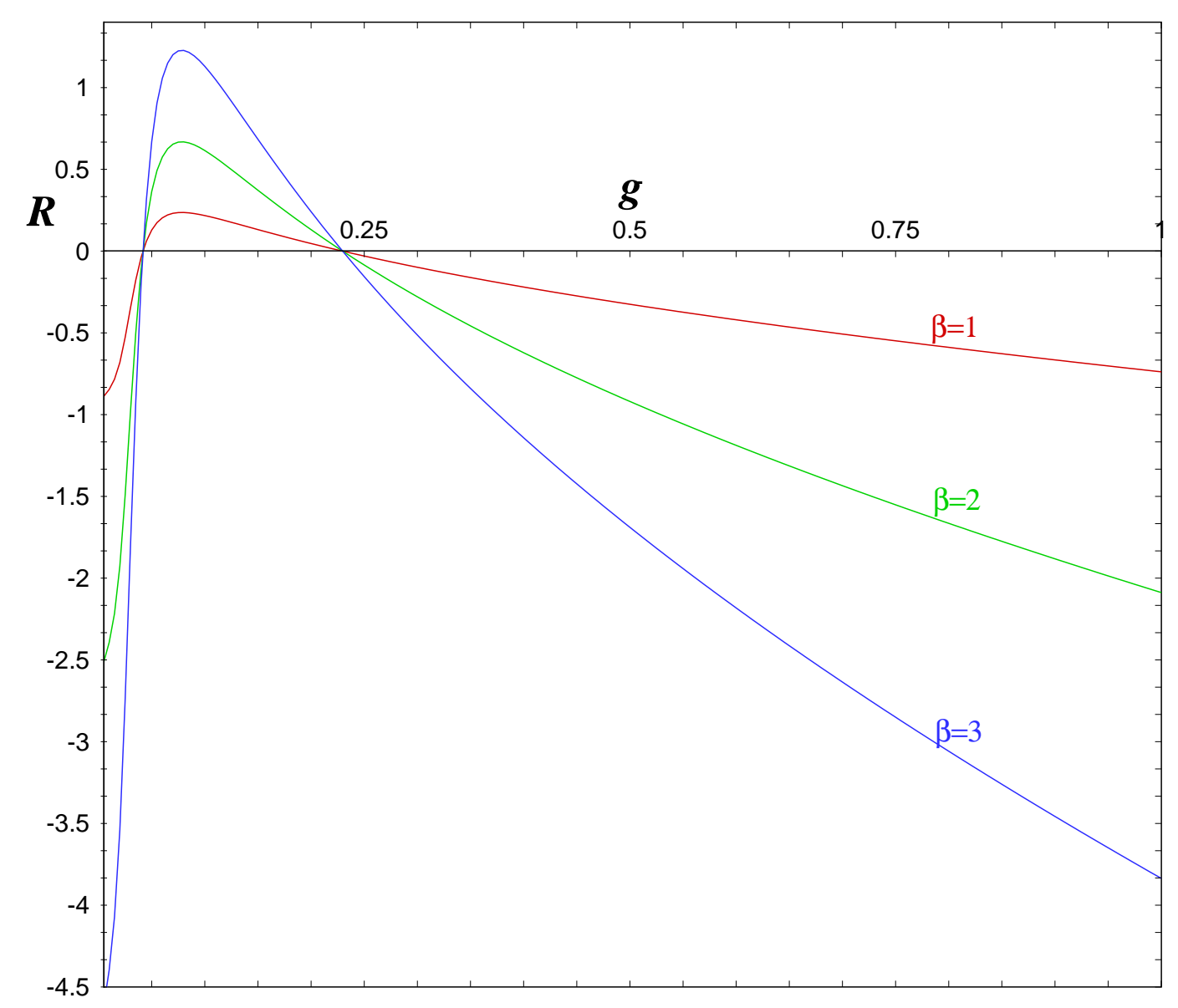

Figure 5: (Color online) The thermodynamic curvature of an ideal 3D HFE gas (nonrelativistic) as a function of the fractional parameter for a fixed value of fugacity ( $z=$ 1.2) and different values of $\beta=1$ [red (lower max) line], 2 [green (middle max) line], 3 [blue (upper max) line]. 


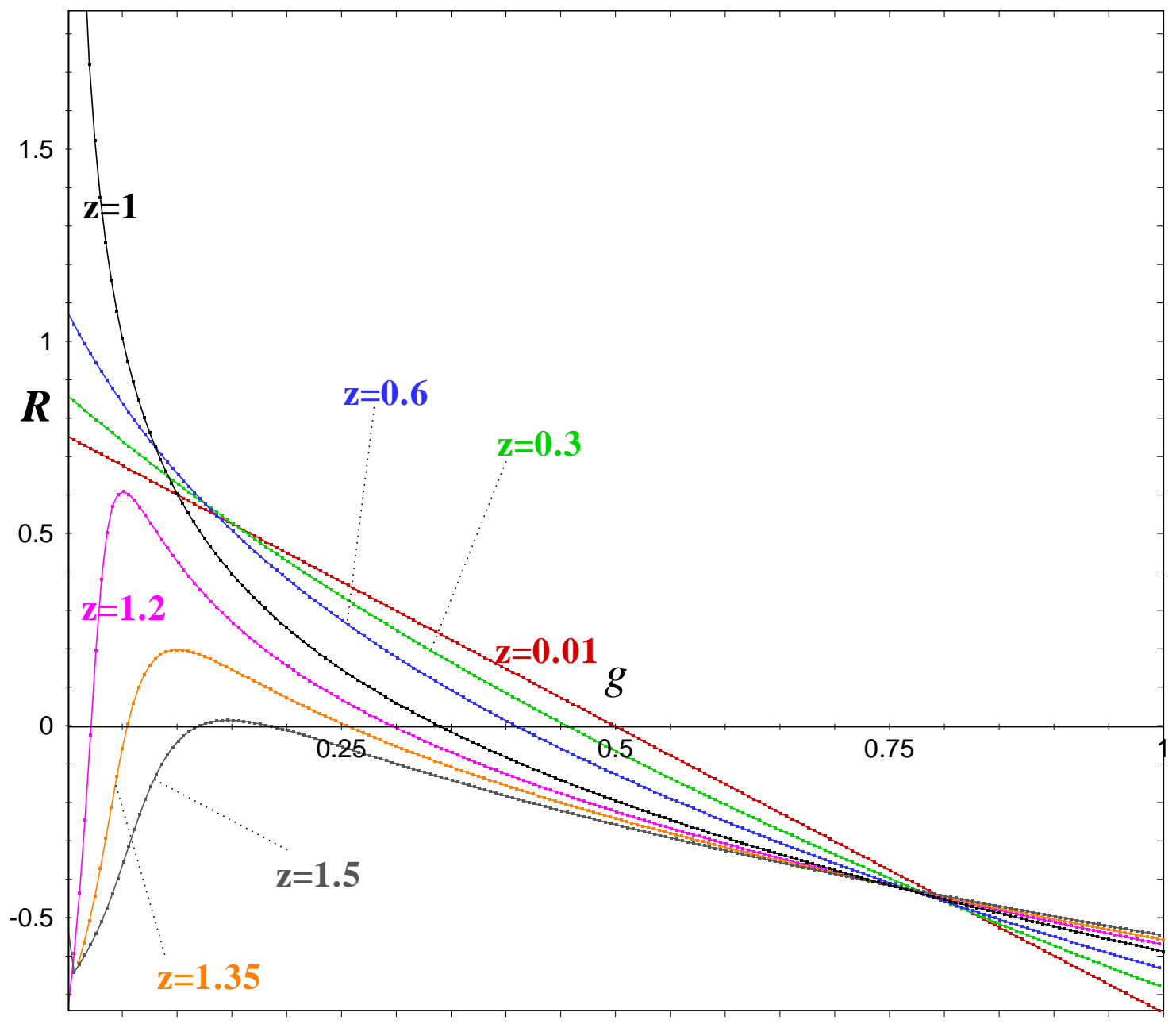

Figure 6: (Color online) The thermodynamic curvature of a 2D HFE gas (ultrarelativistic limit) as a function of fractional parameter for an isotherm $(\beta=1)$. The assumed values of anyon fugacity are $z=0.01$ (red line) , 0.3 (green line), 0.6 (blue line), 1 [black (middle) line], 1.2 (purple line), 1.35 (orange line), 1.5 (gray line). 


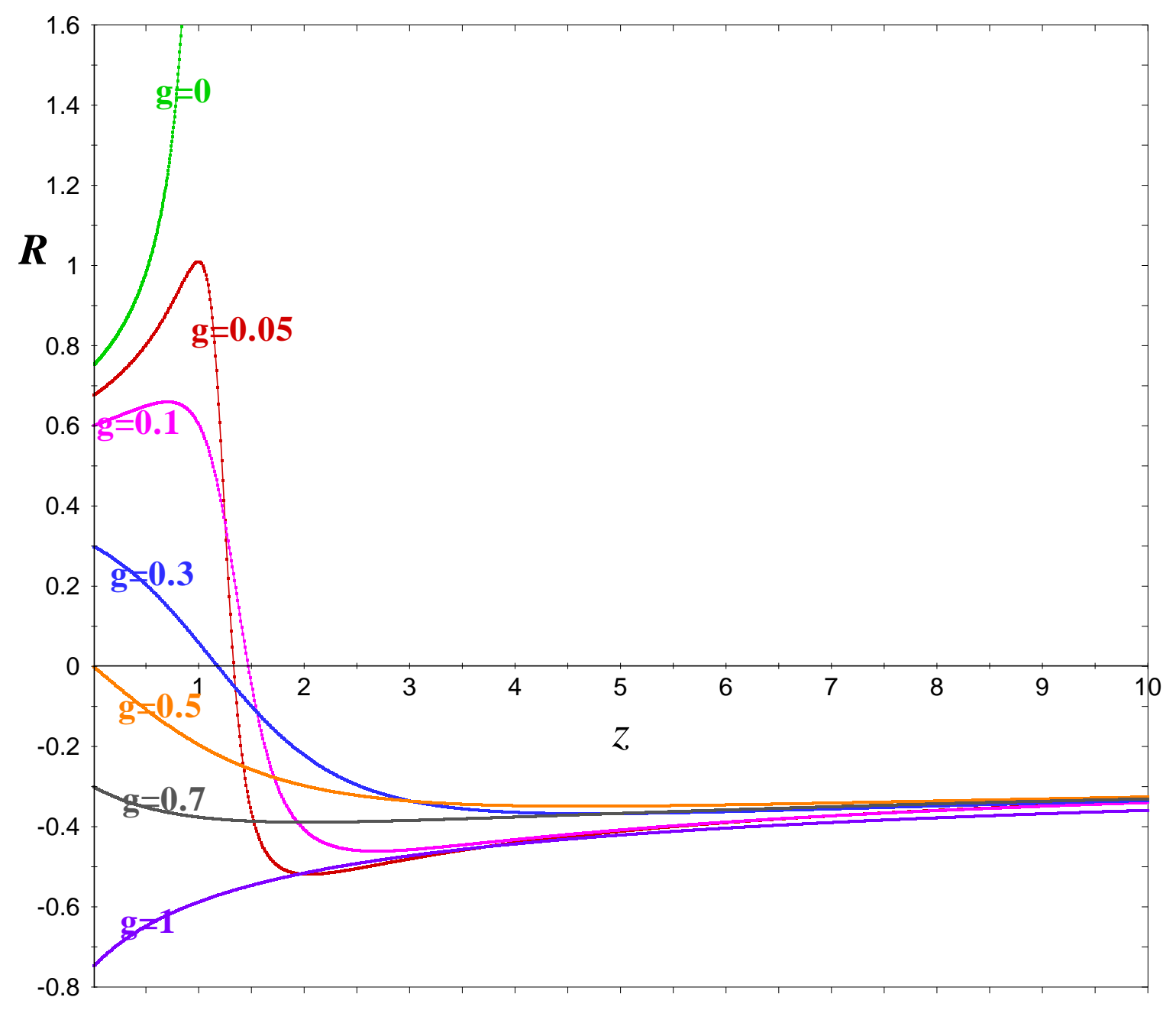

Figure 7: (Color online) The thermodynamic curvature of an ideal 2D ((ultrarelativistic) HFE gas as a function of $z$ for an isotherm $(\beta=1)$. The assumed values of fractional parameter are $g=0$ (green line), 0.05 (red line), 0.1 (purple line), 0.3 (blue line), 0.5 (orange line), 0.7 (black line), 1 (red line). 


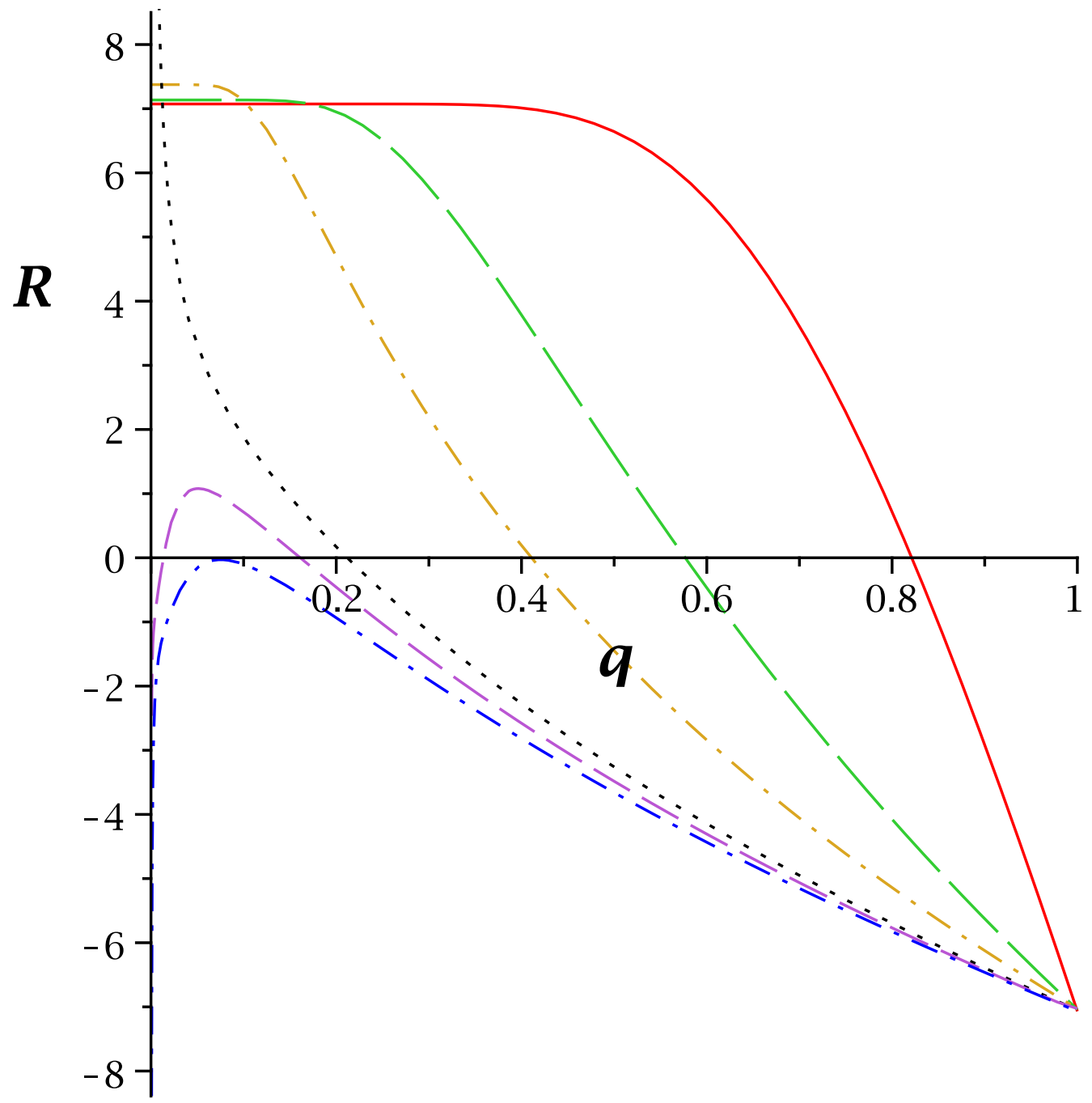

Figure 8: (Color online) The thermodynamic curvature of an ideal 3D Gentile statistic gas as a function of $q$ for an isotherm $(\beta=1)$. The assumed values of fugacity are $z=0.01$ [red (solid) line] , 0.2 [green (long dashed) line], 0.6 [brown (dash dotted) line], 1 [black (dotted) line], 1.2 [violet (dashed) line], 1.3 [blue (lower) line]. 


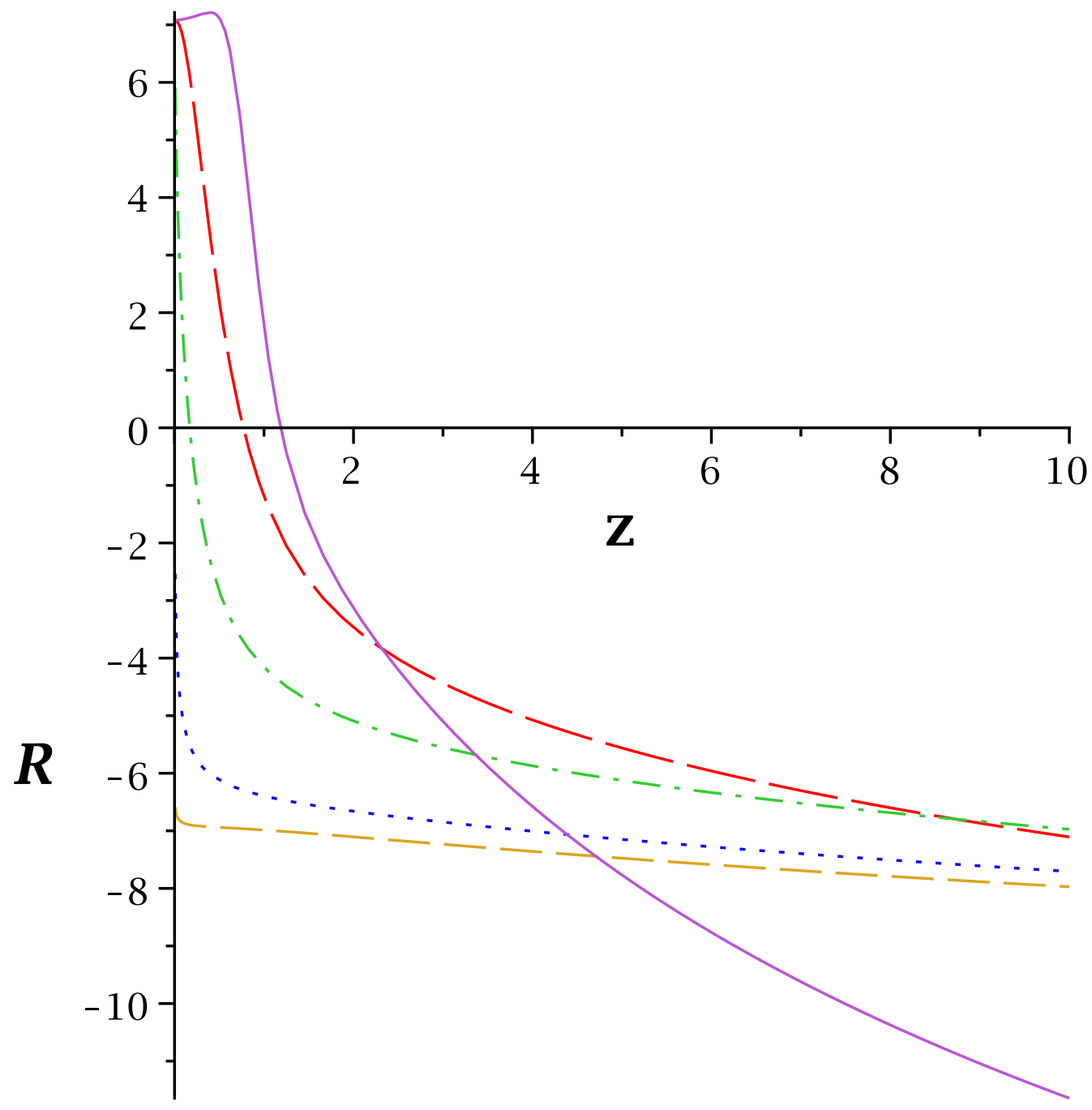

Figure 9: (Color online) The thermodynamic curvature of an ideal 3D Gentile statistic gas as a function of fugacity for an isotherm $(\beta=1)$. The assumed values of the fractional parameter are $q=0.1$ [violet (solid) line], 0.3 [red (long dashed) line], 0.6 [green (dash dotted) line], 0.9 [blue (dotted) line], 1 [brown (dashed) line]. 


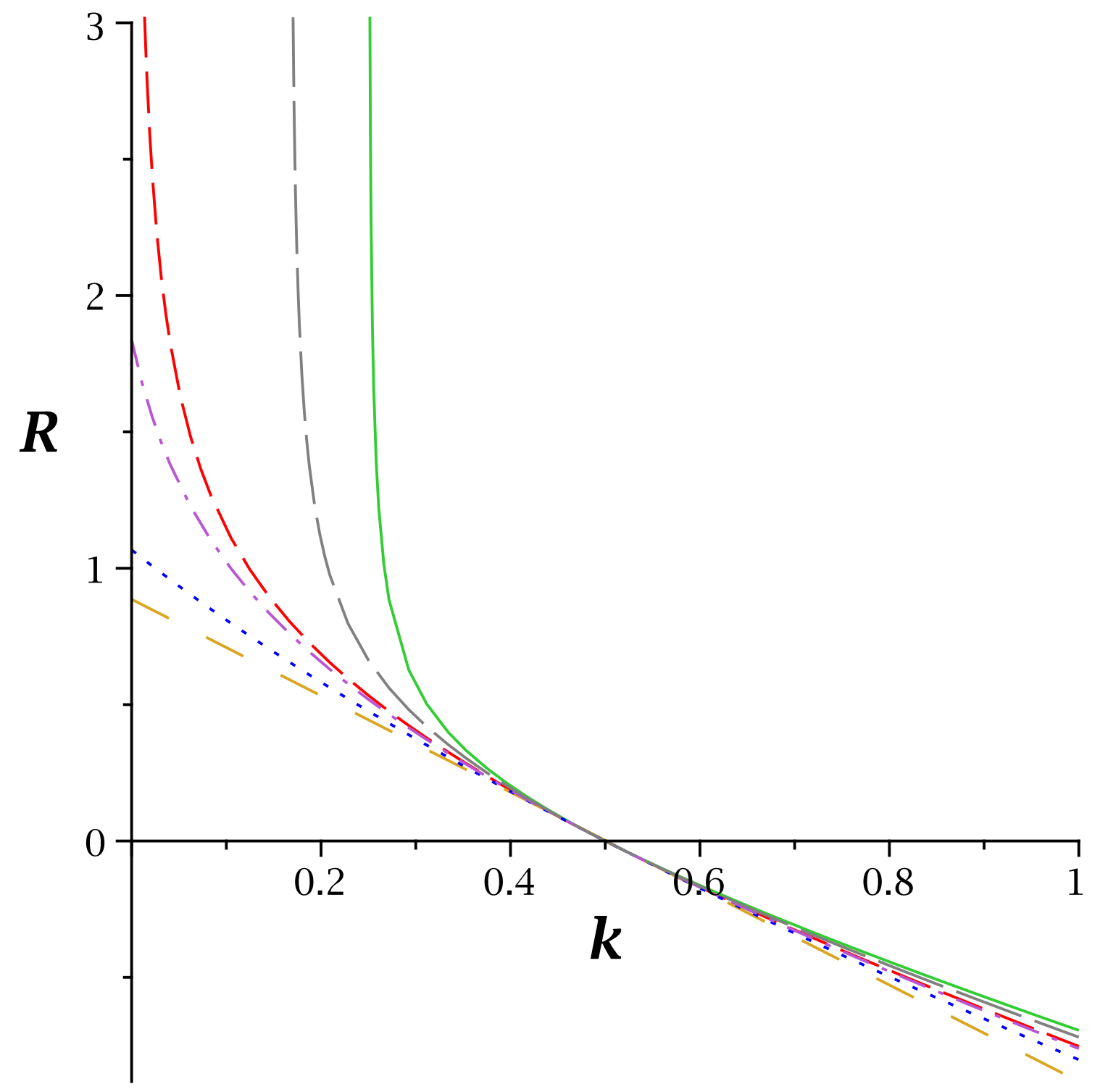

Figure 10: (Color online) The thermodynamic curvature of an ideal 3D PFE gas as a function of $k$ for an isotherm $(\beta=1)$. The assumed values of fugacity are $z=2$ [green (solid) line], 1.5 [gray (long dashed) line], 1 [red (dashed) line], 0.9 [violet (dash dotted) line], 0.5 [blue (dotted) line), 0.01 [brown (lower space dashed) line]. 


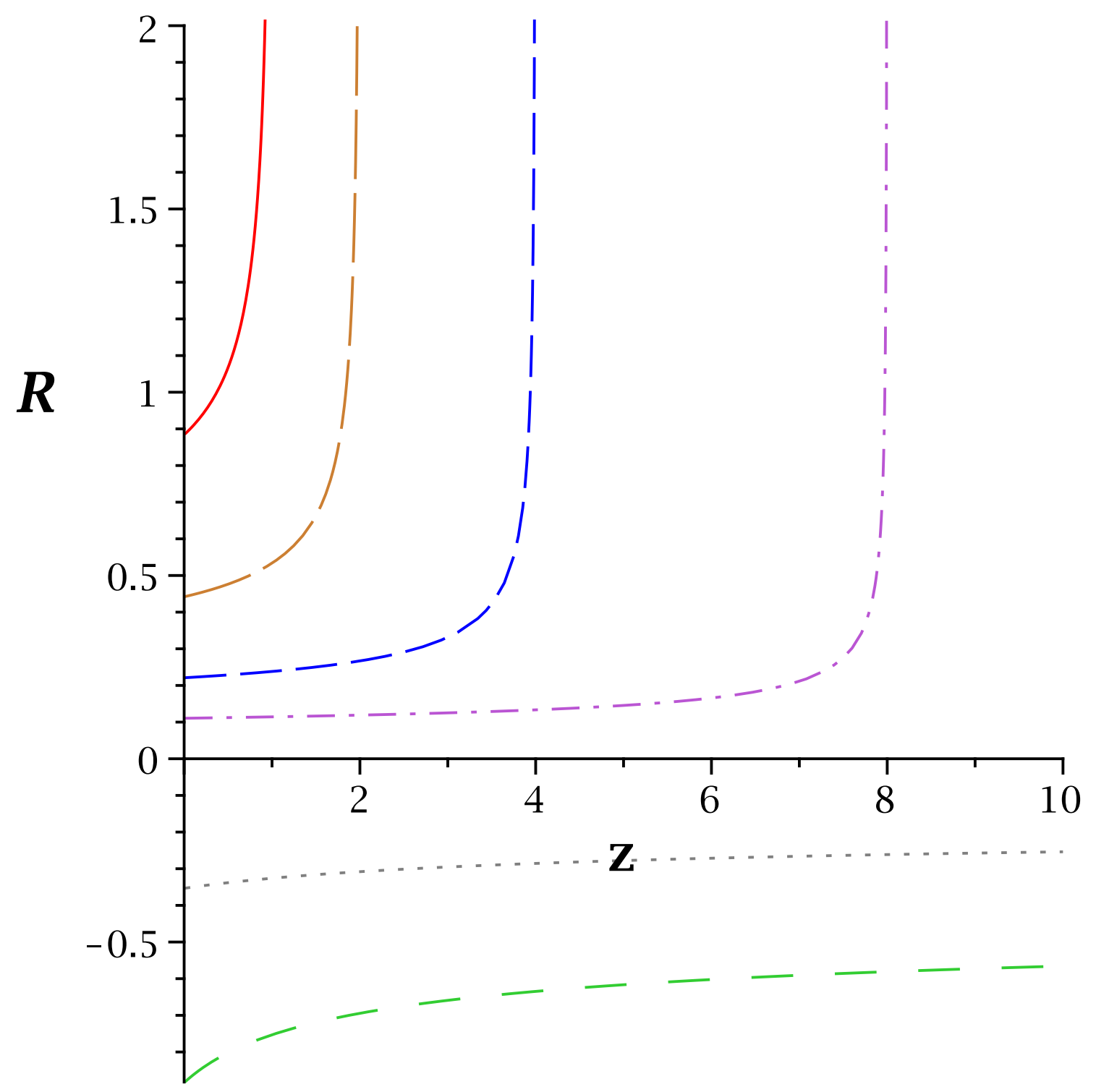

Figure 11: (Color online) The thermodynamic curvature of a 3D ideal PFE gas as a function of fugacity for an isotherm $(\beta=1)$. The assumed values of the fractional parameter are $k=0$ [red (solid) line], $\frac{1}{4}$ [brown (long dashed) line], $\frac{3}{8}$ [blue (dashed) line], $\frac{7}{16}$ [violet (dash dotted) line], 0.7 [gray (dotted) line], 1 [green (lower space dashed) line]. 


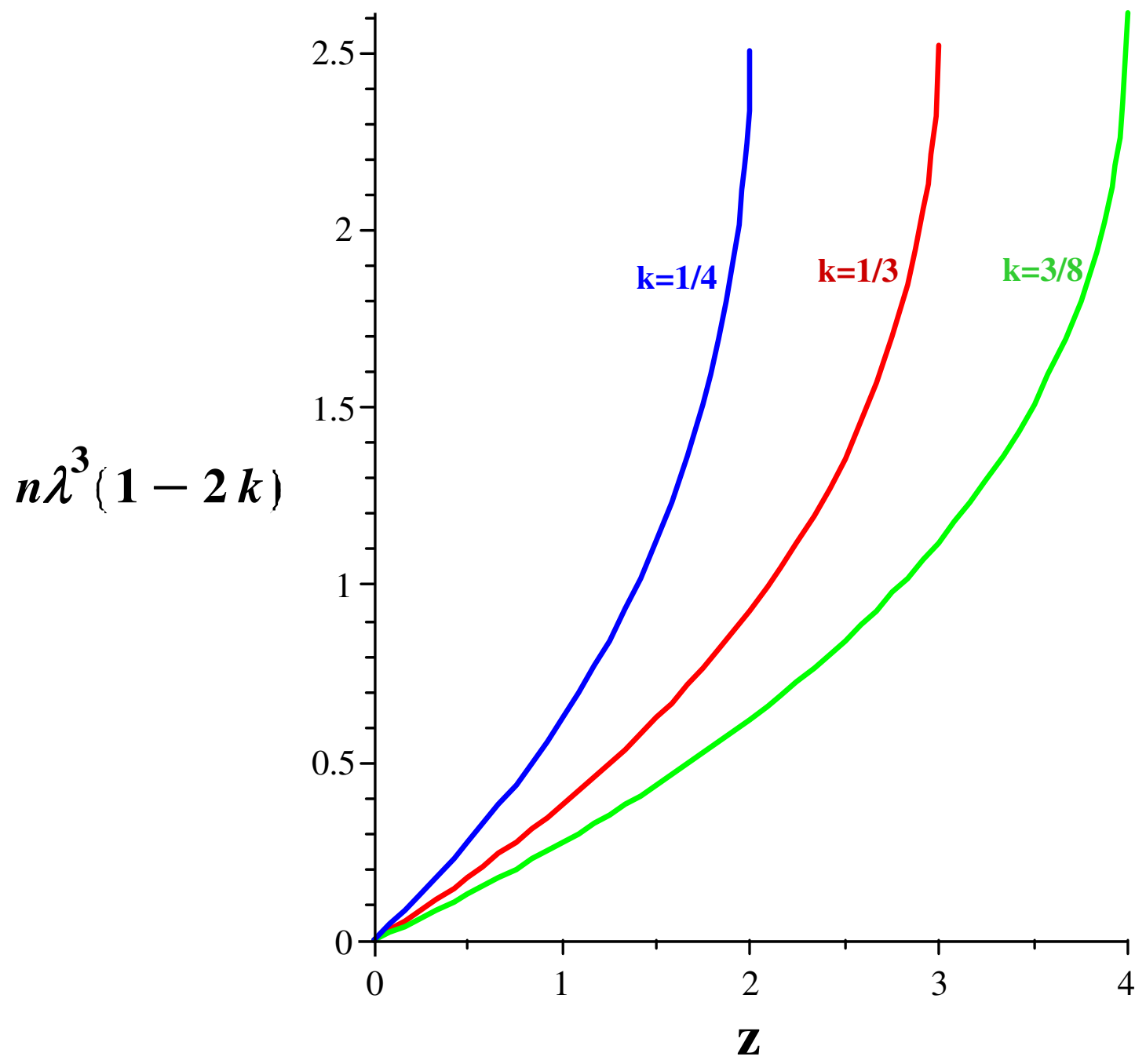

Figure 12: (Color online) $n \lambda^{3}(1-2 k)$ as a function of fugacity for some values of fractional parameter, $k=\frac{1}{4}$ (blue line), $\frac{1}{3}$ (red line), $\frac{3}{8}$ (green line). 